\title{
Proceeding
}

Supplementary Issue: Autumn Conferences of Sports Science. Costa Blanca Sports Science Events, 2-3 November 2018.

Alicante, Spain

\section{Postural evaluation in young skaters: Effect of two proprioceptive training}

\author{
CRISTIANA LUCCHETTI 14 , VINCENZO BIANCALANA ${ }^{1}$, MASSIMO ROSSATO² \\ ${ }^{1}$ Department of Biomolecular Sciences, University of Urbino, Italy \\ ${ }^{2}$ Postural Equip Academy, Venezia, Italy
}

\begin{abstract}
The roller skating involves developing of motor and artistic skills from an early age. The aim was to evaluate the effects that an ocular a podalic proprioceptive training cause to postural control and stability in a sample of young skaters. A total of 25 skaters aged between 9 and $15(12 \pm 2.14)$ divided into two groups: the eyes group (EG) aimed at exercising visual proprioception, the feet group (FG) aimed at exercising podalic proprioception. The participants were subject to through electronic stabilometry in static mode, open (OE) and closed (CE) eyes, in a time T0 (pre-training) and T1 (post-training), without and with skates. The results through the two paired t-test showed that both variables Wz $(\mathrm{TotHz})$ to CE within the EG $(p<.05)$ and Wx $(\mathrm{TotHz})$ to CE within the FG $(p<.05)$ were statistical significance. The unpaired t-test showed that the FG obtained a greater statistical significance than the EG for the VarVit and Wx (TotHz) variables in CE $(p<.05)$ and $\mathrm{Wz}(\mathrm{TotHz})$ in both CE and OE $(p<.05)$. The two-way ANOVA found statistical significance $(p<.05)$ on the Wx (TotHz) variable in open/closed eyes of the FG (T0/T1). We concluded that in roller skating it could be useful to integrate athletic training with a visual proprioceptive training. Key words: Proprioceptive training; Postural evaluation; Young skaters.
\end{abstract}

\section{Cite this article as:}

Lucchetti, C., Biancalana, V., \& Rossato, G. (2019). Postural evaluation in young skaters: Effect of two proprioceptive training. Journal of Human Sport and Exercise, 14(1proc), S105-S114. doi:https://doi.org/10.14198/jhse.2019.14.Proc1.13

Corresponding author. Department of Biomolecular Sciences, University of Urbino, Italy.

E-mail: cristiana.lucchetti@uniurb.it

Supplementary Issue: Autumn Conferences of Sports Science. Costa Blanca Sports Science Events, 2-3 November 2018. Alicante, Spain.

JOURNAL OF HUMAN SPORT \& EXERCISE ISSN 1988-5202

(c) Faculty of Education. University of Alicante.

doi:10.14198/jhse.2019.14.Proc1.13 


\section{INTRODUCTION}

Figure roller skating is a complex sport and the athlete must do accurately the technical elements, interpret the musical base, pay attention to the fluidity and elegance of single motor gesture (Merlo, 2009; Kapoula, 2006). The athletes perform technical gestures and movements increasingly difficult, and are subjected to frequent and rigorous training sessions. The most common problems in this sport are musculoskeletal injuries (acute and / or chronic) that mainly involve the foot, the ankle, the knee, the leg, the hip and the back lumbosacral region (Pecina, 1990). The traumas and the training overload are the main causes of injuries involving skaters (Porter et al. 2007). Stress fractures are the most frequent injuries on junior women skaters (Dubravcic-Simunjak et al., 2003).

The Tonic Postural System controls posture. This system uses the information sent by the proprioceptive, vestibular, and visual sensory systems, in order to organize appropriate responses (Berne et al., 2009; Roll et al., 1991). The Tonic Postural System is a complex system, since it is a cybernetic system, the result of a reflex activity that evolves during growth and regresses with aging. Man needs a proprioceptive system that is able to activate the interaction between the system itself and the environment and memory circuits (engram), in order to maintain the posture in erect biped position (Kramer, \& Sylvester, 2009). The main receptors (proprioceptors) of the Tonic Postural System that regulate the visuo-podalic axis are the visual receptor and the podalic receptor (Bricot, 1999; Roll et al., 1987). Physical activity training aimed at training the proprioceptive system is necessary to ensure that the neuromotor system continues to function efficiently over the years. It also reduces the risk of injuries through the musculoskeletal system strengthening, the coordination and the body perception (Alessandria, \& Pilandieri, 2016; Cordo, 1994; Gagey, \& Weber, 2000).

\section{Hypotheses}

The aim of this study is to evaluate and compare the acute modification of postural control and stability of an ocular proprioceptive training and a podalic proprioceptive training in a sample of young skaters (Biancalana, \& Nart, 2018).

\section{MATERIALS AND METHODS}

\section{Participants}

The study included 31 female skaters with age between 9 and $15(12 \pm 2.14)$. The exclusion criteria were the strabismus, monocular blindness, cardiovascular disease or Central Nervous System dysfunctions, foot congenital malformations, an impossibility to stop the wheels on skate. Four girls with health diseases were excluded and two girls because of a defective skate (Tab. I). All participants' parents signed on informed consent form before starting treatment.

Table 1. Characteristics of the participants in the study. Data are represented using the arithmetic mean (M) and standard deviation (SD)

\begin{tabular}{ccc}
\hline & Participants $(\mathbf{n}=\mathbf{2 5})$ & \\
\hline Age (years) & $12 \pm 2.14$ & $64.2 \pm 7.2$ \\
Weight $(\boldsymbol{K})$ & $42.57 \pm 11.4$ & $32.8 \pm 6.2$ \\
Height $(\mathbf{c m})$ & $151.44 \pm 11.93$ & $22.4 \pm 4.1$ \\
\hline
\end{tabular}

\section{Measures}

The socio-demographic variables (age, sex) and the anthropometric data (weight, height) have been detected through a standardized medical history interview and recorded in a special personal card. 
A battery of tests was carried out to confirm the inclusion criteria and to verify the sample homogeneity:

- Dominant eye test, performed on each eye for three times with a covering cardboard to evaluate the far/ near vision (preferential eye) (Alessandria \& Piladieri, 2016).

- Proximal Point Convergence/PPC, performed using the Lang fixation bar to observe the behaviour of maximum eye muscle activity (Barraud, 1997) and the eyes' convergence.

- Visual acuity test, performed using the Snellen chart to measure visual acuity (Snellen, 1873).

- Astigmatism test, performed using the image of a star with twelve rays, in each ray there are three parallel lines. It's possible to diagnose an astigmatism if the image appears more or less clear to the subject.

- Titmus Stereo Test, stereopsis test performed with polarized glasses using the LEA® symbols (Vision Assessment Corporation, Illinois, USA) This test quickly evaluates the presence of amblyopia, strabismus and binocular acuity problems.

- Stabilometric assessment, performed to evaluate the stabilometric parameters in open and closed eyes condition, in static mode (AFP85 Standards). The recording was performed with Cyber Sabots $®$ instruments (In Tech s.r.l., Marseilles, France). The data were processed using the SabotSoftVib software.

\section{Procedures}

A total of 25 subjects divided into two groups randomly: Eyes Group ( $E G, n=12)$, Feet Group ( $F G, n=13$ ). The members of the EG were subjected to a proprioceptive exercise protocol for eye receptors (ocular proprioceptive training) and the members of the FG were subjected to a proprioceptive exercise protocol for podalic receptors (podalic proprioceptive training) (Figure 1).

Participants of both groups underwent 2 stabilometric evaluations with the aim of evaluating the effect of proprioceptive training on the variables considered, namely: a first, at T0 (prior to proprioceptive training), and then at T1 (following proprioceptive training). In both evaluations, the subjects wore skates whose rollers had previously been locked in order to avoid any oscillations that could have possibly compromised the test result.

The EG undergone a training protocol characterized by Ocular proprioceptive training and a Kinesthetic saturation.

Ocular proprioceptive training session was performed without wearing skates and using instead the Bern's arrow as an aid tool (SprintIT s.r.l., Noale, VE) (Figure 2). The training session, which lasted 25 minutes on the whole, involved the repetition of a cycle of orthopaedic exercises using a Bern's arrow positioned in three different directions (namely: parallel to the floor, with the tip turned forward and the base resting at the halfway point of the nose length; parallel to the floor, with the tip turned forward and the base resting in such a way as to coincide with the eyebrows; perpendicular to the floor, with the base turned forward and the tip resting on the root of the nose). For each position, the subjects were required to fix the central circle with both eyes until succeeding identifying an "X", then to look at the farthest circle, visualizing an upside-down "V", and finally to move the eye from a circle to the another, keeping staring at each of them for 10 seconds' period of time.

The Kinaesthetic saturation involved exercises to be performed while wearing skates, aimed at improving the proximal point of convergence as well as maximizing the work of oculomotor muscles, through the evocation of the convergence reflex. 
The FG underwent a Podalic proprioceptive training session, performed without wearing skates. The training session, which lasted 25 minutes, involved the execution of 5 different proprioceptive gaits: walking on the forefoot and on the heels, walking on the outer side and inside of the foot, walk with rolling foot.

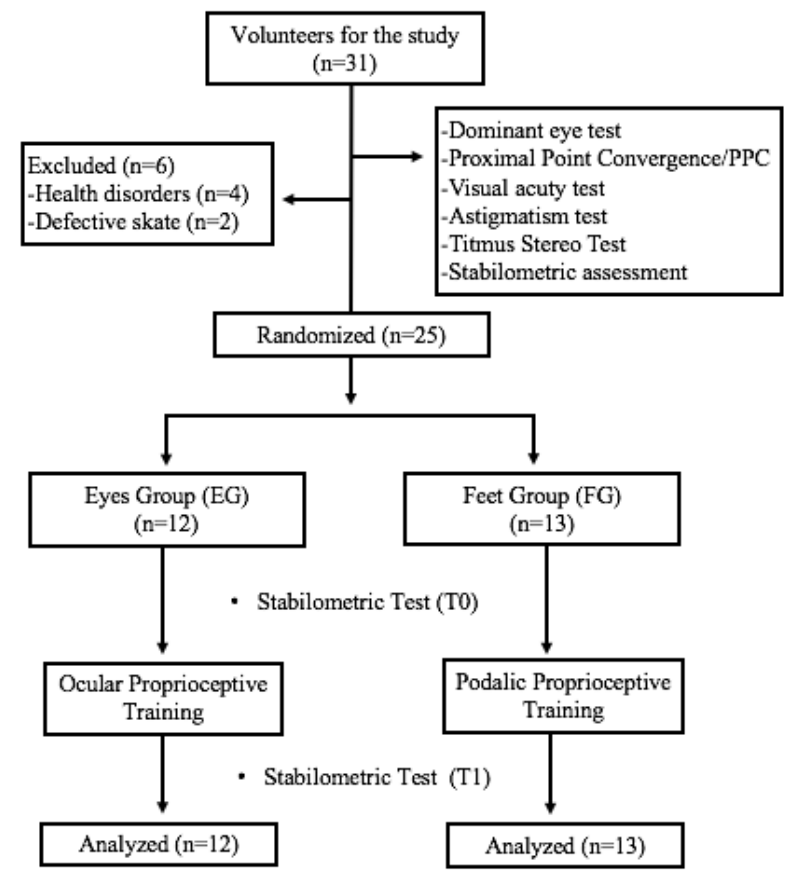

Figure 1. Study design

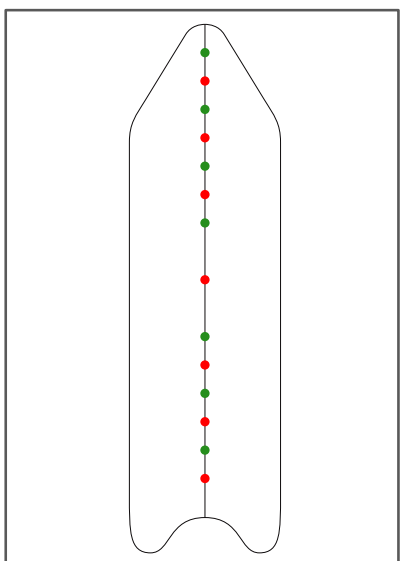

Figure 2. The Bern's arrow

\section{Analysis}

In particular, among the set of parameters acquired with the stabilometric platform, the following ones were considered:

- Surface, which represents the ellipse containing $90 \%$ of the points recorded during the acquisition time in relation to the displacement of the Centre of Pressure (CoP), i.e. the statokinesigram, and expresses the degree of oscillation of the postural tonic system. 
- VarVit, which refers to the average of speed variations recorded, and is closely related to the CoP displacements along the entire length of the statokinesigram. The greater the variation in speed, the more unstable the system, thus requiring adjustments to maintain balance.

$-W x(T o t H z)$, which represents the energy of the oscillations throughout the entire frequency band (from 0 to $20.0 \mathrm{~Hz}$ ), on the $\mathrm{X}$ axis (frontal oscillations).

The variables are calculated using the Fourier transform, which allows performing an analysis of the stabilometric signal by expressing both the power and amplitude of the components. For each variable, the mean (M) and the standard deviation (SD) were calculated, as well as comparing the mean values so identified with the standard values derived from the literature and assumed as a condition of normality (Rossato et al., 2013). As for the statistical analysis, the GraphPadPrism 7 program was used instead. Initially, a two-tailed student's unpaired t-test was performed to check if there were any significant differences between $\mathrm{T} 0$ and $\mathrm{T} 1$ within the EG group and the FG group in open eyes and closed eyes conditions for each of the variables considered. Subsequently, a one-tailed student's unpaired t-test was performed comparing the T1-T0 difference for each variable, both in open eyes and closed eyes condition, in order to verify which of the two groups had benefited most from the proprioceptive training session.

Finally, the ANOVA two-way variance analysis tests were used to check if any changes had occurred within the same group between T0 and T1 and between the open eyes and closed eyes conditions and the posthoc Bonferroni test, in the event that any statistical significance being so detected.

The significance level was set at $p \leq 0.05$ and the confidence interval was set at $95 \%$.

\section{RESULTS}

\section{Paired T-test}

The analysis of the data derived on the effect of proprioceptive training between T0 and T1 in each group and for each of the variables considered highlighted the presence in the EG group of a statistically significant difference $(p=0.0419)$ only for the $\mathrm{Wz}(\mathrm{TotH} z)$ variable in the closed eyes condition, while in the FG group a statistically significant difference was highlighted $(p=0.0472)$ solely for the $W x(T o t H z)$ variable in the closed eyes condition (Figure 3-4).

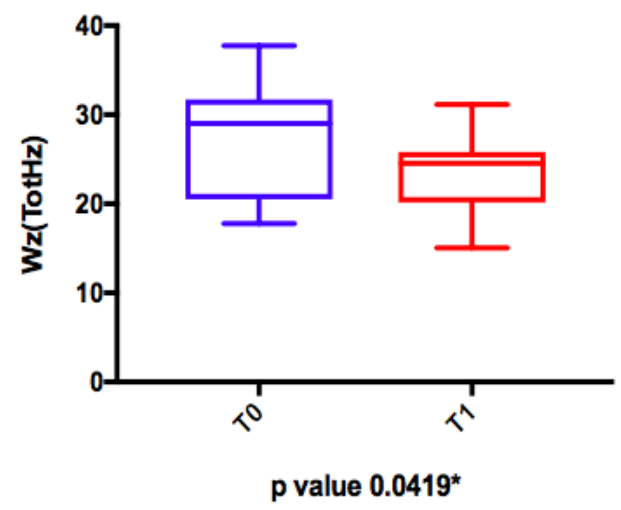

Figure 3. The effects of proprioceptive training between $\mathrm{TO}$ and $\mathrm{T} 1 \mathrm{in}$ the $\mathrm{EG}$, for the $\mathrm{Wz}(\mathrm{TotHz})$ variable, in the closed eyes condition. Data are represented using the arithmetic mean and standard deviation. (significance level $=p \leq 0.05$ ) 


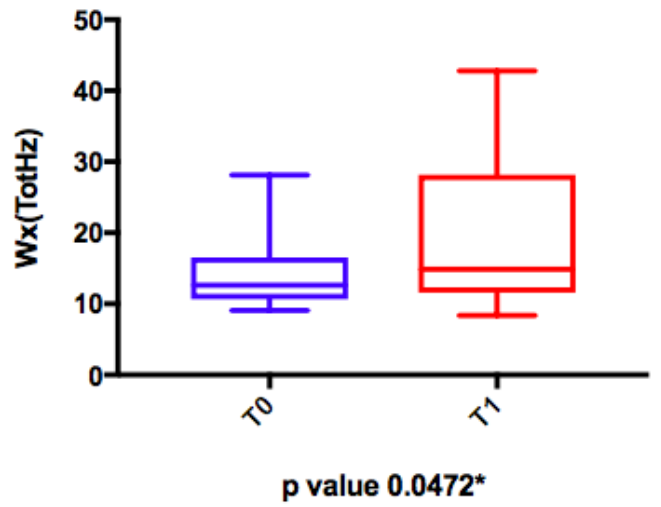

Figure 4. The effects of proprioceptive training between $\mathrm{T0}$ and $\mathrm{T} 1$ in the $\mathrm{FG}$, for the $\mathrm{Wx}(\mathrm{TotHz})$ variable, in the closed eyes condition. Data are represented using the arithmetic mean and standard deviation. (significance level $=p \leq 0.05$ )

\section{Unpaired T-test}

Considering the difference (mean value) between T1 and T0 values in the EG group $(-2,56 \pm 5,04)$ and in the FG group $(1,42 \pm 5,31)$, for the $\mathrm{Wz}(\mathrm{TotHz})$ variable, in the open eyes condition, it has been manifested a statistically significant difference $(p=0.0261)$ (Figure 5$)$.

\begin{tabular}{|c|c|c|c|c|}
\hline Name & EG & Name & FG & \\
\hline 1 & $-3,56$ & 1 & 8,17 & Improvement \\
\hline 2 & $-1,91$ & 2 & $-3,56$ & Decrease \\
\hline 3 & 2,81 & 3 & 6,32 & \\
\hline 4 & $-1,49$ & 4 & $-8,94$ & \\
\hline 5 & $-2,47$ & 5 & 0,24 & \\
\hline 6 & 6,41 & 6 & 9,64 & \\
\hline 7 & $-6,01$ & 7 & $-3,38$ & \\
\hline 8 & $-2,23$ & 8 & 2,34 & \\
\hline 9 & $-5,23$ & 9 & 0,45 & \\
\hline 10 & $-2,63$ & 10 & 3,43 & \\
\hline 11 & $-11,04$ & 11 & 1 & \\
\hline 12 & $-3,43$ & 12 & 5,79 & \\
\hline & & 13 & $-3,08$ & \\
\hline
\end{tabular}

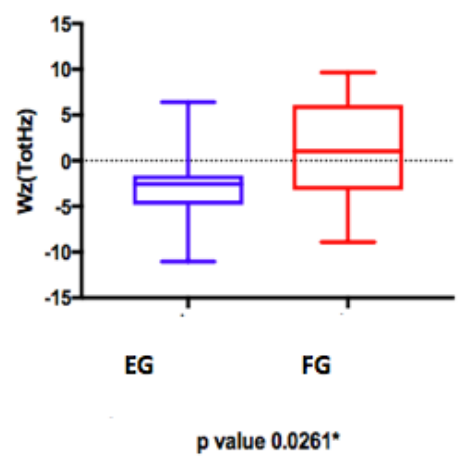

Figure 5. The effects of proprioceptive training to 11 in both groups (EG; FG), for the $\mathrm{Wz}(\mathrm{TotHz}$ ) variable, in the open eyes condition. Data are represented using the arithmetic mean and standard deviation. (significance level $=p \leq 0.05$ )

Considering the difference (mean value) between T1 and T0 values in the EG group $(-33,14 \pm 74,43)$ and in the FG group $(30,28 \pm 95,35)$, for the VarVit variable, in the closed eyes condition, it has been manifested a statistically significant difference $(p=0.0392)$ (Figure 6$)$. 


\begin{tabular}{|c|c|c|c|c|}
\hline Name & EG & Name & FG & \\
\hline 1 & $-30,6$ & 1 & 14,7 & Improvement \\
\hline 2 & $-48,49$ & 2 & $-11,27$ & Decrease \\
\hline 3 & $-27,89$ & 3 & $-4,81$ & \\
\hline 4 & 44,27 & 4 & 73,9 & \\
\hline 5 & $-48,51$ & 5 & $-11,29$ & \\
\hline 6 & 51,23 & 6 & 182,4 & \\
\hline 7 & $-35,59$ & 7 & $-78,3$ & \\
\hline 8 & 60,5 & 8 & $-22,31$ & \\
\hline 9 & $-22,8$ & 9 & $-12,3$ & \\
\hline 10 & $-12,11$ & 10 & 50,05 & \\
\hline 11 & $-120,1$ & 11 & $-21,11$ & \\
\hline 12 & $-207,6$ & 12 & $-32,5$ & \\
\hline & & 13 & 266,5 & \\
\hline & & & & \\
\hline
\end{tabular}

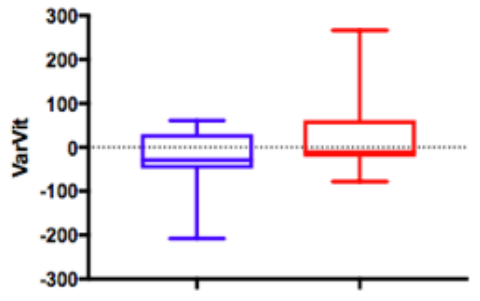

EG

FG

Figure 6. The effects of proprioceptive training to $T 1$ in both groups (EG; FG), for the VarVit variable, in the closed eyes condition. Data are represented using the arithmetic mean and standard deviation. (significance level $=p \leq 0.05$ )

Considering the difference (mean value) between T1 and T0 values in the EG group $(-1,64 \pm 2,86)$ and in the FG group $(5,32 \pm 8,67)$, for the $W x(T o t H z)$ variable, in the closed eyes condition, it has been manifested a statistically significant difference $(p=0.0072)$ (Figure 7).

\begin{tabular}{|c|c|c|c|c|}
\hline Name & EG & Name & FG & \\
\hline 1 & $-1,533$ & 1 & 1,6 & Improvement \\
\hline 2 & $-2,303$ & 2 & $-2,31$ & Decrease \\
\hline 3 & $-1,06$ & 3 & 2,53 & \\
\hline 4 & 2,033 & 4 & 0,36 & \\
\hline 5 & $-2,948$ & 5 & $-0,969$ & \\
\hline 6 & 3 & 6 & 26,5 & \\
\hline 7 & $-3,047$ & 7 & 14,66 & \\
\hline 8 & 0,92 & 8 & $-0,036$ & \\
\hline 9 & $-3,11$ & 9 & $-0,69$ & \\
\hline 10 & $-0,805$ & 10 & 14,29 & \\
\hline 11 & $-2,94$ & 11 & 1,84 & \\
\hline 12 & $-7,899$ & 12 & $-0,01$ & \\
\hline & & 13 & 11,36 & \\
\hline
\end{tabular}

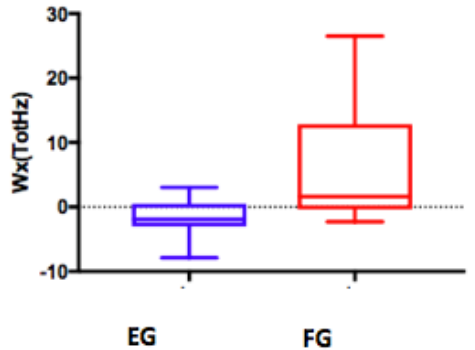

p value $0.0072^{* *}$

Figure 7. The effects of proprioceptive training to $\mathrm{T} 1$ in both groups (EG; FG), for the $\mathrm{Wx}(\mathrm{TotHz})$ variable, in the closed eyes condition. Data are represented using the arithmetic mean and standard deviation. (significance level $=p \leq 0.05$ )

Considering the difference (mean value) between T1 and T0 values in the EG group $(-3,35 \pm 5,04)$ and in the FG group $(1,92 \pm 7,39)$, for the $\mathrm{Wz}(\mathrm{TotHz})$ variable, in the closed eyes condition, it has been manifested a statistically significant difference $(p=0.0252)$ (Figure 8$)$. 


\begin{tabular}{|c|c|c|c|c|}
\hline Name & EG & Name & FG & \\
\hline 1 & $-2,58$ & 1 & 0,39 & Improvement \\
\hline 2 & $-4,43$ & 2 & $-2,96$ & Decrease \\
\hline 3 & $-0,61$ & 3 & 0,55 & \\
\hline 4 & 1,75 & 4 & 0,48 & \\
\hline 5 & $-2,72$ & 5 & 2,52 & \\
\hline 6 & 6,69 & 6 & 7,62 & \\
\hline 7 & $-7,91$ & 7 & 3,71 & \\
\hline 8 & $-5,74$ & 8 & $-2,99$ & \\
\hline 9 & $-3,49$ & 9 & 0,72 & \\
\hline 10 & $-0,19$ & 10 & 21,3 & \\
\hline 11 & $-8,94$ & 11 & 3,7 & \\
\hline 12 & $-12,03$ & 12 & $-11,69$ & \\
\hline & & 13 & 1,61 & \\
\hline
\end{tabular}

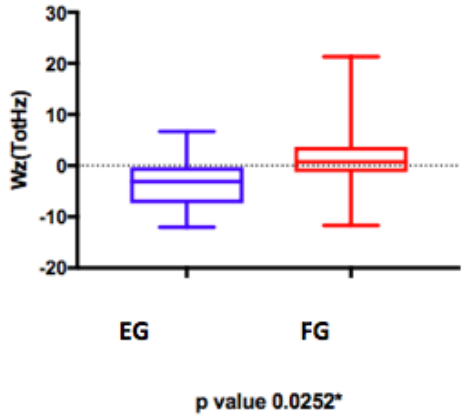

Figure 8. The effects of proprioceptive training to T1 in both groups (EG; FG), for the $\mathrm{Wz}(\mathrm{TotHz})$ variable, in the closed eyes condition. Data are represented using the arithmetic mean and standard deviation. (significance level $=p \leq 0.05$ )

Considering the differences (mean value) between T1 and T0 values in each group (EG; FG) for the VarVit variable in the open eyes condition, Surface in open and closed eyes condition, $\mathrm{Wx}(\mathrm{TotHz})$ in the open eyes condition, $\mathrm{Wy}(\mathrm{TotHz})$ in open and closed eyes condition, it hasn't been manifested a statistically significant difference $(p \geq 0.05)$.

\section{Two-way ANOVA}

The analysis of data, only in the FG group, for the $\mathrm{Wx}(\mathrm{TotHz})$ variable, in the closed eyes condition, has shown a statistically significant difference $(p=0.0210)$, between T0 mean value $(14,47 \pm 5,24)$ and T1 mean value $(19,78 \pm 11,81)$ (Figure 9).

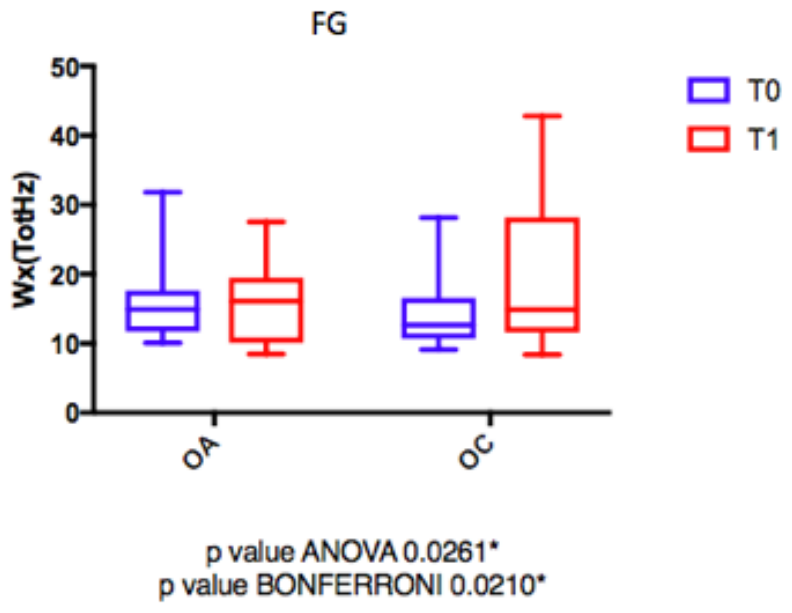

Figure 9. The effects of proprioceptive training between T0 and T1 in the FG, for the $\mathrm{Wx}(\mathrm{TotHz})$ variable, in open and closed eyes condition. Data are represented using the arithmetic mean and standard deviation. (significance level $=p \leq 0.05$ )

\section{DISCUSSION}

The aim of this study was to assess and compare the postural changes and the acute stability during the acute phase of ocular proprioceptive training and podalic proprioceptive training in a sample of young women skaters. From the results on the variables considered, it emerged that at T1, for the VarVit variable, the EG 
group reduced the average of the recorded speed variations compared to the FG group, thus showing that the former obtained a greater benefit from the proprioceptive training session. As for the $W x(\mathrm{TotHz})$ variable in the FG group, a lower effect of proprioceptive training was highlighted, with a significant increase in the energy of the post-training oscillations compared to the EG group. In the EG group, for the $W z(T o t H z)$ variable, at $\mathrm{T} 1$ there was a significant reduction in the energy of the oscillations in favour of a better postural control compared to the FG group, whose average increased instead. As for the Surface and Wy(TotHz) variables, no significance was highlighted. This would seem to indicate that, during the acute phase, proprioceptive training did not cause any change - in area in which the CoP moves - to the energy of the oscillations on the $Y$ axis (sagittal oscillations). Compared to the $W x(T o t H z)$ and $W z(T o t H z)$ variables, the $W y(T o t H z)$ variable during the acute phase does not undergo any significant changes at the end of the proprioceptive training session; this could be due to the skate boot, which limits the flexion and extension movements of the ankle, thus preventing any significant improvements and/or worsening of the oscillations on the sagittal plane.

The FG group showed an increase in the oscillations on the $X$ axis and a worse performance in qualitative terms compared to the EG group for the VarVit, $\mathrm{Wx}(\mathrm{TotHz})$ and $\mathrm{Wz}(\mathrm{TotHz})$ variables. This performance gap could result from a reduced sensitivity of the podalic and articular receptors of the ankle determined by the skate boot, which, by blocking the ankle, does not allow the foot to move inside it (Kavounoudias et al., 1998). The podalic proprioceptive training could actually have destabilized, during the acute phase, the compensations put in place by the postural tonic system. In the EG group, the decreased oscillation energy on the vertical axis and the significantly better performance compared to the FG group, for the VarVit, $\mathrm{Wx}(\mathrm{TotHz})$ and $\mathrm{Wz}(\mathrm{TotHz})$ variables, could suggest the evocation, during the acute phase, of certain rearrangements of the postural tonic system through ocular proprioceptive training.

\section{CONCLUSION}

It can conclude that in roller skating it could be useful to integrate athletic training with a visual proprioceptive training in order to improve postural control and reduce the risk of accidents (Riva et al, 2001).

\section{REFERENCES}

Alessandria, M., \& Pilandieri, A. (2016). Guida di posturologia clinica. Modelli logico- funzionali dell'organizzazione del sistema posturale. Tecniche Nuove - Milano.

Berne, R.M., Levy, M.N., Koeppen, B.M., \& Stanton, B.A. (2009). Fisiologia. C.E.A.Casa Editrice Ambrosiana, Milano.

Biancalana, V., \& Nart, A. (2018). Postural analysis from foot to the centre of mass with ledpodolaser. Science Movement and Health, 18(1), 25-30.

Bricot, B. (1999). La riprogrammazione posturale globale. Statipro - Marseille.

Cordo, P., Carlton, L., Bevan, L., Carlton, M., \& Kerr, G. K. (1994). Proprioceptive coordination of movement sequences: role of velocity and position information. Journal of Neurophisiology, 71(5), 1848-1861. https://doi.org/10.1152/in.1994.71.5.1848

Dubravcic-Simunjak, S., Pecina, M., Kuipers, H., Moran, J., \& Haspl, M. (2003). The Incidence of Injures in Elite Junior Figure Skaters. The American Journal of Sports Medicine, 31(4), 511-517. https://doi.org/10.1177/03635465030310040601

Gagey, P. M., \& Weber, B. (2000). Posturologia. Regolazione e perturbazioni della stazione eretta. Marrapese Editore - Roma. 
Kapoula, Z., \& Le, T. T. (2006). Effects of distance and gaze position on postural stability in young and old subjects. Experimental Brain Research, 173(3), 438-445. https://doi.org/10.1007/s00221-006$0382-1$

Kavounoudias A, Roll R, Roll JP (1998) The plantar sole is a 'dynamometric map' for human balance control. Neuroreport, 9: 3247-3252. https://doi.org/10.1097/00001756-199810050-00021

Kramer, P, \& Sylvester, A. (2009). Bipedal form and locomotor function: understanding the effects of size and shape on velocity and energetics. PaleoAnthropology, 238, 251. https://doi.org/10.4207/PA.2009.ART32

Lee, D. N., \& Aronson, E. (1974). Visual proprioceptive control of standing in human infants. Perception \& Psychophysics, 15(3), 529-532. https://doi.org/10.3758/BF03199297

Manzoli, M. V., Rota, I., Facchin, A., Maffioletti, S. (2011). La stereopsi globale e locale. Quaderni acp, $18(1), 27-31$.

Merlo, A. (2009). Pattinaggio artistico individuale libero. FIHP.

Pecina, M., Bojanic, I., \& Dubravcic, S. (1990). Stress fractures in figure skaters. The American Journal of Sports Medicine, 18, 277-279. https://doi.org/10.1177/036354659001800310

Peterka, R. J. (2002). Sensorimotor integration in human postural control. Journal of Neurophysiology, 88(3), 1097-1118. https://doi.org/10.1152/jn.2002.88.3.1097

Porter, E. B., Young, C. C., Niedfeldt, M. W., \& Gottschlich, L. M. (2007). Sport-Specific Injuries and Medical Problems of Figure Skaters. Wisconsin Medical Journal, 106(6), 330-334.

Riva, D., Botta, M., Trevisson, P., Minoletti, R., \& Venturin, N. (2001). Postural strategies in figure skating. Sport \& Medicina.

Roll, J. P., Roll, R., Kavounoudias, A. (2002). Cutaneos afferentes from human plantar sole contribute to body awareness. Pubmed-Neurore Port, 13(15), 19.

Roll, J. P., \& Roll, P. (1987). Kinesthesic and motor effects of extraocular muscle vibration in man, Eye movements. Amsterdam J.K O'Regan\&A, Lev-Schoen, 57-58.

Roll, R., Roll, J. P., \& Rossato, M. (2017). Stabilometria e riabilitazione. Seminario di posturologia. Sprintit, Noale.

Roll, R., Velay, J. I., \& Roll, J. P. (1991). Eye and neck proprioceptive message contibute to the spatial coding retinal input in visually-oriented activity. Exp Brain Res, 85, 423-31. https://doi.org/10.1007/BF00229419

Rossato, M., Bourgeois, P., \& Ouaknine, M. (2013). Stabilometry standard guidelines 2011-2013 during clinical practice. Marrapese editore - Roma.

Snellen, H. (1873). Probebuchstaben zur Bestimmung der Sehschärfe. Berlin - 4e. Ausg.

\section{(9) $(\mathbb{0} \Theta \Theta$}

This work is licensed under a Attribution-NonCommercial-NoDerivatives 4.0 International (CC BY-NC-ND 4.0). 